\title{
Semantic Decision Procedures for Some Relevant Logics
}

\author{
Ross BRADY \\ Philosophy Program, La Trobe University \\ Ross.Brady@latrobe.edu.au \\ Received by Greg Restall \\ Published July I, 2003 \\ http://www.philosophy.unimelb.edu.au/ajl/2003 \\ (c) 2003 Ross Brady
}

Abstract: This paper proves decidability of a range of weak relevant logics using decision procedures based on the Routley-Meyer semantics. Logics are categorized as F-logics, for those proved decidable using a filtration method, and U-logics, for those proved decidable using a direct (unfiltered) method. Both of these methods are set out as reductio methods, in the style of Hughes and Cresswell. We also examine some extensions of the U-logics where the method fails and infinite sequences of worlds can be generated.

In Relevant Logics and their Rivals [12, pages 399-406], Richard Routley (as he was then known) purported to have established the decidability of a range of weak relevant logics. He first claimed to have proved decidability for the system B, on pp. 4OI-2, using a filtration method upon the Routley-Meyer semantics, which yielded the finite model property for в. After warning that the method would by no means extend to all the systems with semantic postulates given in Chapter 4 and 5 of [12], he extended the result to the postulate Raaa, for the axiom $A \&(A \rightarrow B) \rightarrow B$, the postulate Raa*a, for $A \rightarrow \sim A \rightarrow \sim A$, and also to the postulate, if Rabc then $\operatorname{Rac}^{*} b^{*}$, for $A \rightarrow \sim B \rightarrow$. B $\rightarrow \sim A$. There are also a couple of less significant postulates given. Routley then examined the use of a second filtration, but realized its shortcomings in establishing postulate P2 - if $a \leq b$ and Rbcd then Racd - for the finite model. He briefly examined a third filtration, realized that it would not yield a decidability result without deductive closures on the worlds of the finite models, and hence suggested using operational semantics instead of the Routley-Meyer semantics.

However, Routley had erred in two places in his first filtration. Fortunately for those of us interested in weak relevant logics and Routley-Meyer-style semantics, his second filtration can still be used and his results can be revived and extended using the simplified semantics of Priest and Sylvan [Io] and Restall 
[II], which has subsequently become available. The object of $\$ 3$ of this paper is to show how this is to be done. In the process, I do not want to detract from what has otherwise been an outstanding volume, of which all of us in the field make heavy use.

It must also be said that Fine had first proved the decidability of weak relevant logics without $\mathrm{A} \rightarrow \mathrm{B} \rightarrow \mathrm{B} \rightarrow \mathrm{C} \rightarrow \mathrm{A} \rightarrow \mathrm{C}$ and $\mathrm{A} \rightarrow \mathrm{B} \rightarrow \mathrm{C} \rightarrow$ $A \rightarrow$.C $\rightarrow B$ in [6], pp. 365-8, using his own semantics which embraces both theories and prime theories. The above postulate $\mathbf{P 2}$ simplifies to - (ii) if $t \leq u$ then $(t \cdot v) \leq(u \cdot v)$ [6, page 348] - which enables the decidability proof to go through without anything resembling the simplified semantics of Priest, Sylvan and Restall.

Moreover, a different range of systems have been shown decidable using proof-theory in Brady [3,4,5], viz. the contraction-less logics, DW, Tw and RW, by using Gentzen systems based on the work of Dunn [I, pages 38I-39I] and Giambrone [7]. This suggests that there may be a semantic method, based on a Routley-Meyer semantics, for establishing the same result, which might then extend to other systems. We will show this in $\$ 5$ of the paper, indicating what goes wrong with some of these extensions.

\section{The Logics}

We present axiomatizations for the main logics referred to in this paper: $\mathbf{B}, \mathbf{B x}$, BC, DW, DWX, DWC, DJ, DK, DL, D, DC, RBC, TW, TWx, TWC and RW, together with their disjunctive rule extensions. We take as primitives: $\sim, \&, \vee, \rightarrow$, and we consider the following axioms, rules and meta-rule, and the class of logics constructed using them, in Figures I and 2. (Each of the subtracted axioms and rules are redundant in the respective system.)

For any logic $L$, let $\mathrm{L}^{\mathrm{d}}$ be $\mathrm{L}+\mathrm{MR}$. For each of the listed logics $\mathrm{L}$ without $\mathrm{AI} 2, \mathrm{~L}={ }_{\mathrm{L}}^{\mathrm{d}}$, i. e., they have the same set of theorems. (See Slaney [I3] and [I4] for this result.)

We distinguish two kinds of logics for the purposes of this paper: An F-logic is one of the following: $B^{\mathrm{d}}($ or $\mathrm{B}), \mathrm{DW}^{\mathrm{d}}$ (or DW), DJ ${ }^{\mathrm{d}}\left(\right.$ or DJ) $, \mathrm{DK}^{\mathrm{d}}, \mathrm{DL}^{\mathrm{d}}, \mathrm{D}^{\mathrm{d}}, \mathrm{DC}^{\mathrm{d}}$, $\mathbf{R B C}^{\mathrm{d}}$. These will used in $\$_{3}$ for filtrations and in $\$_{4}$ for the reductio method based on these.

A U-logic is one of the following : $\mathbf{B}^{\mathrm{d}}($ or $\mathrm{B}), \mathbf{B X}^{\mathrm{d}}, \mathrm{BC}^{\mathrm{d}}, \mathrm{DW}^{\mathrm{d}}\left(\right.$ or Dw), $\mathrm{DW} \mathrm{x}^{\mathrm{d}}$, DwC ${ }^{\mathrm{d}}, \mathrm{Tw}^{\mathrm{d}}$ (or Tw), $\mathrm{TwX}^{\mathrm{d}}, \mathrm{TwC}^{\mathrm{d}}, \mathrm{RW}^{\mathrm{d}}$ (or Rw). These will be used in $\$ 5$ for the reductio method without filtrations. Since simplified semantics are used throughout, disjunctive rules are always required.

\footnotetext{
'We use 'RBC' to represent the logic R, without the two B-axioms, AI 6 and AI7, but with the other half of classicality, R5.
} 


\begin{tabular}{|c|c|}
\hline AI & $A \rightarrow A$ \\
\hline A2 & $A \& B \rightarrow A$ \\
\hline A3 & $A \& B \rightarrow B$ \\
\hline A4 & $(A \rightarrow B) \&(A \rightarrow C) \rightarrow . A \rightarrow B \& C$ \\
\hline A5 & $A \rightarrow A \vee B$ \\
\hline А 6 & $B \rightarrow A \vee B$ \\
\hline A7 & $(A \rightarrow C) \&(B \rightarrow C) \rightarrow . A \vee B \rightarrow C$ \\
\hline A 8 & $A \&(B \vee C) \rightarrow(A \& B) \vee(A \& C)$ \\
\hline A9 & $\sim A \rightarrow A$ \\
\hline AIO & $\mathrm{A} \rightarrow \sim \mathrm{B} \rightarrow . \mathrm{B} \rightarrow \sim \mathrm{A}$ \\
\hline AII & $(A \rightarrow B) \&(B \rightarrow C) \rightarrow . A \rightarrow C$ \\
\hline AI 2 & $A \vee \sim A$ \\
\hline $\mathrm{AI} 3$ & $A \rightarrow \sim A \rightarrow \sim A$ \\
\hline AI 4 & $(A \rightarrow . A \rightarrow B) \rightarrow . A \rightarrow B$ \\
\hline AI5 & $A \&(A \rightarrow B) \rightarrow B$ \\
\hline AI 6 & $\mathrm{~A} \rightarrow \mathrm{B} \rightarrow . \mathrm{B} \rightarrow \mathrm{C} \rightarrow . \mathrm{A} \rightarrow \mathrm{C}$ \\
\hline AI 7 & $\mathrm{~A} \rightarrow \mathrm{B} \rightarrow . \mathrm{C} \rightarrow \mathrm{A} \rightarrow . \mathrm{C} \rightarrow \mathrm{B}$ \\
\hline AI 8 & $A \rightarrow . A \rightarrow B \rightarrow B$ \\
\hline RI & $A, A \rightarrow B \Rightarrow B$ \\
\hline R2 & $A, B \Rightarrow A \& B$ \\
\hline R3 & $\mathrm{A} \rightarrow \mathrm{B}, \mathrm{C} \rightarrow \mathrm{D} \Rightarrow \mathrm{B} \rightarrow \mathrm{C} \rightarrow \mathrm{A} \rightarrow \mathrm{D}$ \\
\hline R4 & $\mathrm{A} \rightarrow \sim \mathrm{B} \Rightarrow \mathrm{B} \rightarrow \sim \mathrm{A}$ \\
\hline $\mathrm{R} 5$ & $\sim A, A \vee B \Rightarrow B$ \\
\hline MRI & If $A \Rightarrow B$ then $C \vee A \Rightarrow C \vee B$ \\
\hline
\end{tabular}

Figure r: Axioms, Rules and Meta-Rule

\section{The Simplified Semantics}

Priest and Sylvan [ı] introduced a simplified version of the Routley-Meyer semantics for the logic $\mathrm{B}^{+}$and then $\mathrm{B}$, the essential feature being the removal of the defined ordering relation ' $\leq$ ' for the reduced semantics and the use of the following special truth-condition for evaluating entailments $A \rightarrow B$ at the base world T, viz.

- $\mathrm{I}(\mathrm{A} \rightarrow \mathrm{B}, \mathrm{T})=\mathrm{T}$ iff, for all $\mathrm{b} \in \mathrm{K}$, if $\mathrm{I}(\mathrm{A}, \mathrm{b})=\mathrm{T}$ then $\mathrm{I}(\mathrm{B}, \mathrm{b})=\mathrm{T}$.

The usual Routley-Meyer truth-condition applies for $A \rightarrow B$ for worlds $a \neq T$.

Then, Restall [II] extended this simplified semantics to a wide range of relevant and other logics. However, he did not use the special truth-condition for $A \rightarrow B$, but instead added the semantic postulate, $R T a b \Leftrightarrow a=b$, which simplifies some of the other postulates, together with the corresponding soundness and completeness arguments. For our purposes, it is Restall's form of the semantics that works best and so we set it out for our logics of interest. We start with the logic $\mathbf{B}^{\mathrm{d}}$, which has the same theorems as $\mathbf{B}$. 


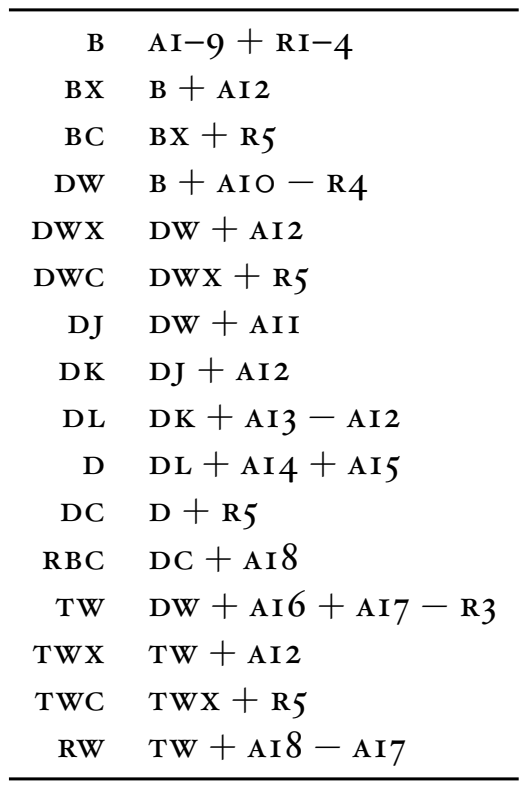

Figure 2: Systems

A в model structure (вms) consists of the concepts $\langle T, K, R, *\rangle$, where $\mathrm{K}$ is a set of worlds, the base world $\mathrm{T} \in \mathrm{K}, \mathrm{R}$ is a 3-place relation on $\mathrm{K}$, and $*$ is a I-place function on $\mathrm{K}$, subject to the semantic postulates: for $\mathrm{a}, \mathrm{b} \in \mathrm{K}$ :

PI $a^{* *}=a$.

P2 $\mathrm{RTab} \Leftrightarrow \mathrm{a}=\mathrm{b}$.

A B-model is a Bms with valuation $v$, which assigns a truth-value $T$ or $F$ to each sentential variable for each world. Each valuation is uniquely extended to an interpretation I by induction on formulae, as follows: for $a \in K$, sentential variable $p$, and formulae $A, B$ :

(i) $I(p, a)=v(p, a)$.

(ii) $\mathrm{I}(\sim A, \mathrm{a})=\mathrm{T}$ iff $\mathrm{I}\left(A, \mathrm{a}^{*}\right)=\mathrm{F}$.

(iii) $\mathrm{I}(\mathrm{A} \& \mathrm{~B}, \mathrm{a})=\mathrm{T}$ iff $\mathrm{I}(\mathrm{A}, \mathrm{a})=\mathrm{T}$ and $\mathrm{I}(\mathrm{B}, \mathrm{a})=\mathrm{T}$.

(iv) $\mathrm{I}(\mathrm{A} \vee \mathrm{B}, \mathrm{a})=\mathrm{T}$ iff $\mathrm{I}(\mathrm{A}, \mathrm{a})=\mathrm{T}$ or $\mathrm{I}(\mathrm{B}, \mathrm{a})=\mathrm{T}$.

(v) $\mathrm{I}(\mathrm{A} \rightarrow \mathrm{B}, \mathrm{a})=\mathrm{T}$ iff, for all $\mathrm{b}, \mathrm{c} \in \mathrm{K}$, if $\operatorname{Rabc}$ and $\mathrm{I}(\mathrm{A}, \mathrm{b})=\mathrm{T}$ then $\mathrm{I}(\mathrm{B}, \mathrm{c})=\mathrm{T}$.

A formula $A$ is true in a B-model iff $I(A, T)=T$, and $A$ is valid in a Bms iff $A$ is true in every $\mathrm{B}$-model on that $\mathrm{Bms}$. $A$ is valid in the simplified semantics for $\mathrm{B}$ iff $A$ is valid in every Bms. 
TheOREM I For formulae $A$, if $A$ is a theorem of $B$ (or $B$ ) then $A$ is valid in the simplified semantics for $\mathbf{B}$.

Proof The usual soundness theorem follows as in [12], but with P2 simplifying the proof of the Entailment Lemma (Lemma 4.2 on page 302) for reduced modelling. For MRI, see Relevant Logics and their Rivals [I2, pages 336-7], or Priest and Sylvan [IO, pages 22O-I].

Theorem 2 For formulae $A$, if $A$ is valid in the simplified semantics for $\mathbf{B}$ then $A$ is a theorem of ${ }_{B}{ }^{d}$ (or $B$ ).

Proof The completeness theorem is generally standard [I2], but with two essential differences. First, the canonical base world $T_{B}$ is constructed so that each rule of ${ }_{B}{ }^{d}$ is closed in it, with help from MRI. (This construction was used in Brady [2, pages 252-256], with quantifiers.) Second, to prove $R_{B} T_{B} a b \Leftrightarrow$ $a=b$ in the canonical model structure, Restall introduces (for $\mathbf{B}^{+}$) [II, page 490], a copy of $T_{B}$, called $T_{B}^{\prime}$, which has the same members as $T_{B}$, but satisfies the above equivalence for $a, b \in K_{B}$. $R_{B} a b c$ is then defined normally for $a \neq$ $T_{B}^{\prime}$. Then, in proving $I_{c}(A \rightarrow B, a)=T$ iff $A \rightarrow B \in a$, for the canonical interpretation $I_{c}$, we need to consider the special case, $a=T_{B}^{\prime}$. However, for $B$, with negation, we also need a copy of $T_{B}^{*}$, viz. $T_{B}^{* \prime}$, so as to satisfy $\mathrm{PI} \cdot{ }^{2} R_{B} T_{B}^{* \prime} a b$ is just defined as $R_{B} T_{B}^{*} a b, T_{B}^{* \prime}$ and $T_{B}^{*}$ being regarded as distinct elements of $\mathrm{K}_{\mathrm{B}}$.

We extend these soundness and completeness results to the F-logics and Ulogics, defined in \$r. Figure 3 lists the semantic postulate corresponding to each additional axiom and rule.

Theorem 3 For formulae $A$, if $A$ is a theorem of one of the F-logics or $U$ logics $L$ then $A$ is valid in the simplified semantics for $L$.

Proof Restall [II, pages 484-6] showed soundness for AII, AI 4, AI5, AI6, AI7 and AI8. After introducing an extra semantic primitive, ' $\leq$ ', with postulates and valuation condition below, Restall [II, pages 506-7], showed soundness for AIO, AI 2 and AI3. Soundness for A5 follows easily. ' $\leq$ ' is a 2-place relation on $\mathrm{K}$, satisfying the following: For $a, b \in K$, and sentential variables $p$,

$$
\begin{aligned}
& \text { PI3 } a \leq b \Rightarrow b^{*} \leq a^{*} \\
& \text { PI4 } a \leq b \& \text { Rbcd } \Rightarrow \text { Racd } 3 \\
& \text { vC If } a \leq b \text { and } v(p, a)=T \text { then } v(p, b)=T .
\end{aligned}
$$

The theorem, 'if $a \leq b$ and $I(A, a)=T$ then $I(A, b)=T$ ', then follows by induction on formulae $A$ [12, page 302].

\footnotetext{
${ }^{2}$ Such an addition as $\mathrm{T}_{\mathrm{B}}^{* \prime}$ was used by Giambrone and Meyer [8, pages II-I2].

${ }^{3}$ Restall breaks this up into two cases: $a=T$ and $a \neq T$, but this does not appear to be needed here.
} 


\begin{tabular}{|c|c|c|c|}
\hline \multicolumn{2}{|c|}{ Additional Axioms and Rule } & \multicolumn{2}{|c|}{ Corresponding Postulate } \\
\hline AIO & $\mathrm{A} \rightarrow \sim \mathrm{B} \rightarrow . \mathrm{B} \rightarrow \sim \mathrm{A}$ & P3 & $\operatorname{Rabc} \Rightarrow \operatorname{Rac}^{*} b^{*}$ \\
\hline AII & $(A \rightarrow B) \&(B \rightarrow C) \rightarrow A \rightarrow C$ & P4 & $\operatorname{Rabc} \Rightarrow \exists x \in K, \operatorname{Rab} x \& \operatorname{Raxc}$ \\
\hline $\mathrm{AI} 2$ & $A \vee \sim A$ & $\mathrm{P} 5$ & $\mathrm{~T}^{*} \leq \mathrm{T}$ \\
\hline AI3 & $A \rightarrow \sim A \rightarrow \sim A$ & $\mathrm{p} 6$ & $\begin{array}{l}\text { (i) } \operatorname{Raa}^{*} a \text {, for } a \neq T \\
\text { and (ii) } T^{*} \leq T\end{array}$ \\
\hline AI 4 & $(\mathrm{~A} \rightarrow . \mathrm{A} \rightarrow \mathrm{B}) \rightarrow . \mathrm{A} \rightarrow \mathrm{B}$ & P7 & $R a b c \Rightarrow \exists x \in K, \operatorname{Rab} x \& R x b c$ \\
\hline AI 5 & $A \&(A \rightarrow B) \rightarrow B$ & $\mathrm{p} 8$ & Raaa \\
\hline AI 6 & $\mathrm{~A} \rightarrow \mathrm{B} \rightarrow \mathrm{B} \rightarrow \mathrm{C} \rightarrow \mathrm{A} \rightarrow \mathrm{C}$ & P9 & $\begin{array}{l}\operatorname{Rab} z \& \operatorname{Rzcd} \Rightarrow \\
\quad \exists x \in \mathrm{K}, \operatorname{Rac} x \& \operatorname{Rbxd}\end{array}$ \\
\hline AI 7 & $\mathrm{~A} \rightarrow \mathrm{B} \rightarrow \mathrm{C} \rightarrow \mathrm{A} \rightarrow \mathrm{C} \rightarrow \mathrm{B}$ & PIO & $\begin{array}{l}\operatorname{Rab} z \& \operatorname{Rzcd} \Rightarrow \\
\quad \exists x \in \mathrm{K}, \operatorname{Rbcx} \& \operatorname{Raxd}\end{array}$ \\
\hline AI 8 & $\mathrm{~A} \rightarrow \mathrm{A} \rightarrow \mathrm{B} \rightarrow \mathrm{B}$ & PII & $R a b c \Rightarrow R b a c$ \\
\hline $\mathrm{R} 5$ & $\sim A, A \vee B \Rightarrow B$ & PI2 & $\mathrm{T} \leq \mathrm{T}^{*}$ \\
\hline
\end{tabular}

[When $\mathrm{AI} 2$ and $\mathrm{R} 5$ are included, replace $\mathrm{P} 6$ (ii) and PI 2 by $\mathrm{T}=\mathrm{T}^{*}$ and simplify $\mathrm{P} 6$ to Raa ${ }^{*}$ a.]

Figure 3: Semantic Postulates Corresponding to Axioms and Rules

However, we only require this process for $T^{*} \leq T$ and $T \leq T^{*}$. In which case, PI3 is automatically satisfied, given PI, and PI4 reduces to the two respective forms, given $\mathbf{P} 2$ :

PI4A $\mathrm{T}^{*} \leq \mathrm{T} \Rightarrow \mathrm{RT}^{*} \mathrm{aa}$

PI4B $T \leq T^{*} \& R T^{*} a b \Rightarrow a=b$

So, in the event that $\mathrm{T}^{*} \leq \mathrm{T}$ is required, i. e. for $\mathrm{AI} 2$ and $\mathrm{AI} 3$, we add:

PI5 RT*aa

and the valuation condition:

vCI If $v\left(p, T^{*}\right)=T$ then $v(p, T)=T$, for all $p$

and, in the event that $T \leq T^{*}$ is required, i. e. for $\mathrm{R}_{5}$, we add the postulate:

PI6 RT*ab $\Rightarrow a=b$

and the valuation condition:

vC2 If $v(p, T)=T$ then $v\left(p, T^{*}\right)=T$, for all $p$

though these are not required on their own, for the indicated logics. 
Theorem 4 For formulae $A$, if $A$ is valid in the simplified semantics for one of the F-logics or U-logics $L$ then $A$ is a theorem of $L$.

Proof Completeness is somewhat more complicated because of the split definition of the canonical relation $R_{L} a b c$, for the logics $L$. We also define $a \leq b$, for $\mathrm{a}, \mathrm{b} \in \mathrm{K}_{\mathrm{L}}$, as $\mathrm{a} \subseteq \mathrm{b}$.

For the logics, $\mathbf{B}^{\mathrm{d}}, \mathbf{B x}^{\mathrm{d}}, \mathbf{B C}^{\mathrm{d}}, \mathbf{D W}^{\mathrm{d}}, \mathbf{D w} \mathbf{x}^{\mathrm{d}}, \mathbf{D W C}^{\mathrm{d}}, \mathbf{D J}^{\mathrm{d}}, \mathbf{D K}^{\mathrm{d}}, \mathbf{D L}^{\mathrm{d}}, \mathbf{D}^{\mathrm{d}}, \mathbf{D C}^{\mathrm{d}}$, $\mathrm{Tw}^{\mathrm{d}}, \mathrm{TwX}^{\mathrm{d}}$ and $\mathrm{TwC}^{\mathrm{d}}$, this is relatively straight-forward with postulates $\mathrm{P}_{3}, \mathrm{P}_{4}$, P6, P7, P8, P9 and PI O requiring additional checking when $\mathrm{a}=\mathrm{T}_{\mathrm{L}}^{\prime}$, as in Restall [II, pages 492-494, 507-8]. For P9 and PIO, we also need to check $b=\mathrm{T}_{\mathrm{L}}^{\prime}$ and $z=\mathrm{T}_{\mathrm{L}}^{\prime}$, but $\mathrm{b}=\mathrm{T}_{\mathrm{L}}$ and $z=\mathrm{T}_{\mathrm{L}}$ do the required work in each case. (Note that P8 is required for $\mathbf{P} 7$, but AI 5 is derivable from AI4 anyway.) Also, we can see that $R_{L} T_{L}^{* \prime} a$ a holds for logics with AI2, since $T_{L}^{*} \subseteq T_{L}$ and $R_{L} T_{L} a a$. Further, for logics with AI2 and $\mathrm{A} 5$, we put $\mathrm{T}_{\mathrm{L}}^{* \prime}=\mathrm{T}_{\mathrm{L}}^{\prime}$ since $\mathrm{T}^{*}=\mathrm{T}$.

However, there is a special definition for $R_{L}$ for logics $L$ containing ar8. We will assume, for the purposes of the logic $\mathrm{RBC}^{\mathrm{d}}$, that $\mathrm{AIO}-\mathrm{I} 5$ and $\mathrm{R} 5$ are included in L, as there is much interlinkage between the various axioms and the rule in re-establishing the postulates. Also, for the purposes of $\mathrm{RW}^{\mathrm{d}}$, we will assume that $\mathrm{AIO}$ is included in $\mathrm{L}$. We define $\mathrm{R}_{\mathrm{L}}$ as:

$$
\mathrm{R}_{\mathrm{L}} \mathrm{T}_{\mathrm{L}}^{\prime} \mathrm{bc} \Leftrightarrow \mathrm{b}=\mathrm{c}, \quad \mathrm{R}_{\mathrm{L}} \mathrm{aT} \mathrm{T}_{\mathrm{L}}^{\prime} \mathrm{c} \Leftrightarrow \mathrm{a}=\mathrm{c}, \quad \mathrm{R}_{\mathrm{L}} \mathrm{ab} \mathrm{T}_{\mathrm{L}}^{\prime} \Leftrightarrow \mathrm{a}=\mathrm{b}^{*}
$$

$R_{L} a b c$ is then defined normally for $a \neq T_{L}^{\prime}, b \neq T_{L}^{\prime}$ and $c \neq T_{L}^{\prime}$. In checking the postulates, $\mathrm{P} 3$ and $\mathrm{PII}$ are fine, $\mathrm{P} 8$ and the unrestricted $\mathrm{P} 6$ use $\mathrm{T}_{\mathrm{L}}^{* \prime}=\mathrm{T}_{\mathrm{L}}^{\prime}, \mathrm{P} 4$ uses $\mathrm{P} 3$ and $\mathrm{P} 8, \mathrm{P} 7$ uses $\mathrm{P} 6$ and $\mathrm{P} 8$, and $\mathrm{P} 9$ and $\mathrm{PIO}$ both use $\mathrm{P} 3$ and $\mathrm{PII}$.

In checking the proof of ' $I_{\mathcal{c}}(A \rightarrow B, a)=T$ iff $A \rightarrow B \in a$ ', we show that $A \rightarrow B \in a$ iff, for all $b, c \in K_{L}$, if $R_{L} a b c$ and $A \in b$ then $B \in c$. In the case $a \neq \mathrm{T}_{\mathrm{L}}^{\prime}, A \rightarrow B \in \mathrm{T}_{\mathrm{L}}^{\prime}$ iff, for all $\mathrm{b} \in \mathrm{K}_{\mathrm{L}}$, if $A \in \mathrm{b}$ then $\mathrm{B} \in \mathrm{b}$, as shown in [Io,II] for $\mathrm{B}^{+}$. For the case $\mathrm{b}=\mathrm{T}_{\mathrm{L}}^{\prime}$, if $A \rightarrow B \in \mathrm{a}, \mathrm{a}=\mathrm{c}, A \in \mathrm{T}_{\mathrm{L}}^{\prime}$ then $\mathrm{B} \in \mathrm{c}$, since $A \rightarrow . A \rightarrow B \rightarrow B$ is a theorem of $L$ and $a$ is a $T_{L}-L$-theory 4 The converse direction is no problem since $T_{L}$ will always suffice for $T_{L}^{\prime}$. (Also see Restall [II, page ${ }_{485}$.) In case $c=T_{L}^{\prime}$, if $A \rightarrow B \in a, a=b^{*}, A \in b$ then $B \in T_{L}^{\prime}$, since $\sim \mathrm{B} \rightarrow . \mathrm{A} \rightarrow \mathrm{B} \rightarrow \sim \mathrm{A}$ is a theorem.

\section{The Filtration Method}

We follow Relevant Logics and their Rivals [12, pages 399-403] in setting up Routley's first filtration and show up the two defects in the argument. We then move onto the second filtration, but employing the simplified semantics of $\$ 2$ for the systems indicated. Generally, the filtration method finitizes the set of worlds of a model by setting as equivalent worlds ones that evaluate a certain finite set $X$ of formulae the same way. This set $X$ is generally the set of subformulae of a given formula under test, closed under the negation of unnegated formulae. The idea here is to show that the logic concerned has the finite

\footnotetext{
${ }^{4}$ For the definition of a T - L-theory, see [12, page 306].
} 
model property, i. e. that each non-theorem can be falsified in a finite model. This yields decidability for the logic by a standard argument [I2, pages 4OI-2].

For an F-logic L, we define an appropriate closure $X$ of a formula $A$ as the set of all subformulae of $A$ closed under the condition: if $B$ is a member of $X$ with its principal connective not being a negation then $\sim B$ is a member of $X$. We consider a Routley-Meyer model $\langle T, O, K, R, *, \leq, v\rangle$, where $K$ is a set of worlds, $\mathrm{T} \in \mathrm{O}, \mathrm{O} \subseteq \mathrm{K}, \mathrm{R}$ is a -place relation on $\mathrm{K}$, $*$ is a $\mathrm{I}$-place function on $\mathrm{K}$, $\leq$ is a 2-place relation on $\mathrm{K}$, subject to the set of postulates below for the logic $\mathrm{L}$, for $\mathrm{a}, \mathrm{b} \in \mathrm{K}$,

PI $a \leq a$,

P2 $\mathrm{a} \leq \mathrm{b}$ and $\mathrm{Rbcd} \Rightarrow \operatorname{Racd}$,

P3 $a \leq b \Rightarrow b^{*} \leq a^{*}$,

P4 $\mathrm{a}=\mathrm{a}^{* *}$,

P5 $a \in O, R a b c \Rightarrow b \leq c$,

p6 $(\exists x \in$ O)Rxaa,

(plus any extra postulates appropriate to the F-logic L) and $v$ is a valuation assigning $T$ or $F$ to sentential variables $p$ at each world $a \in K$, subject to the condition: for sentential variables $p$, if $a \leq b$ and $v(p, a)=T$ then $v(p, b)=T$.

We define, for worlds $a, b \in K$ and appropriate closure $X, a=x$ for $(\forall B \in X)(I(B, a)=I(B, b))$. We call such $a$ and $b X$-equivalent. We note that $a=x b$ iff $a^{*}=x b^{*}$. We define $X$-equivalence classes $\check{a}(X)$ as $\{b \in K: a=x b$, generated by the element $a$. We call these classes $X$-worlds and form the set K of such worlds, i. e. $\check{K}(X)=\{\check{a}(X): a \in K\}$. We will standardly leave off ' $(\mathrm{X})$ ' to simplify terminology. Further, $\check{\mathrm{K}}$ is finite, as each $\mathrm{X}$-world represents a valuation of the set of formulae in $X$, and so if $X$ has $n$ formulae then there are at most $2^{n}$ valuations of $X$ and hence at most $2^{n}$ members of $\check{K}$.

We go on to define a (finite) $X$-model $\check{M}$ in $\check{K}$ which is Routley's first filtration: $\langle\check{T}, \check{O}, \check{K}, \check{R}, \check{*}, \check{\leq}, \check{v}\rangle$. $\check{T}$ is already given, $\check{a}^{\check{*}}={ }_{\mathrm{df}}\left(\mathrm{a}^{*}\right)^{\check{2}}$, and $\mathrm{O}, \check{\mathrm{R}}, \check{\leq}$, and $\check{v}$ are given as follows:

- $\check{a} \in$ Ǒ iff $(\forall B \in X) I(B \rightarrow B, a)=T$,

- $\check{R}$ ǎb̌c iff $(\forall B, C \in X)(B \rightarrow C \in X \& I(B \rightarrow C, a)=T \& I(B, b)=T \Rightarrow$ $\mathrm{I}(\mathrm{C}, \mathrm{c})=\mathrm{T})$,

- $\check{a} \check{c} \check{b}$ iff $(\forall B \in X)(I(B, a)=T \Rightarrow I(B, b)=T)$,

- $\check{v}(p, \check{a})=T$ iff $v(p, a)=T$ and $p \in X$. 
The first problem is that the definition of ă $\in$ O does not seem independent of the choice of the element $a$ in a.. One could have $a=x b, I(B \rightarrow B, a)=T$ and $\mathrm{I}(\mathrm{B} \rightarrow \mathrm{B}, \mathrm{b})=\mathrm{F}$, for some $\mathrm{B} \in \mathrm{X}$ such that $\mathrm{B} \rightarrow \mathrm{B} \notin X$, since the formula $B$ can be evaluated at worlds outside of ă.

The second problem is in checking $\mathrm{P} 5$, we again seem to need $\mathrm{B} \rightarrow \mathrm{B} \in X$, when $B \in X$. We let $B \in X$ and $I(B, b)=T$, and, since $\check{a} \in \mathrm{O}, \mathrm{I}(\mathrm{B} \rightarrow \mathrm{B}, \mathrm{a})=\mathrm{T}$. Since $\check{R}$ ǎb̌c we would need $B \rightarrow B \in X$ to derive $I(B, c)=T$, as required for $\check{b} \check{\leq} \check{c}$.

One could try replacing the definition of $\check{a} \in$ O by $(\forall B \rightarrow B \in X) \mathrm{I}(\mathrm{B} \rightarrow$ $B, a)=T$. This solves the first problem but $P 5$ still does not follow. One could additionally try putting $(\forall B \in X)(B \rightarrow B \in X \& I(B, a)=T \Rightarrow I(B, b)=T)$ for $\check{a} \check{\leq} \check{b}$. This then solves the two problems but creates a new one for $\mathbf{P} 2$. Clearly, we cannot add the condition 'if $B \in X$ then $B \rightarrow B \in X$ ' without losing the finitude of $X$.

We could try to remove Ǒ by using reduced models, i. e. by putting $\check{O}=\check{T}$, but $\mathrm{P} 5$ is still a problem for the original definition of $\stackrel{\check{\leq}}{\leq}$ and $\mathrm{P} 2$ is still a prob-

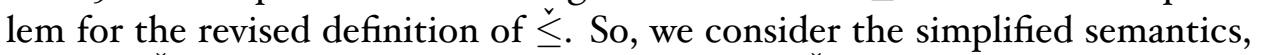
without $\check{O}$ and $\check{\leq}$, but retaining the defintion of $\check{R}$. This does overcome the problems with the initial postulates, and does provide finite modelling for such systems as $B^{d}$ and $D w^{d}$, as set out in [I2, pages 4OO-3], but does not cope with any of the existential postulates, such as $\mathrm{P} 4$ and $\mathrm{P} 7$ of $\$ 2$, which would yield systems such as $\mathrm{DJ}^{\mathrm{d}}$ and $\mathrm{D}^{\mathrm{d}}$.

So, we move on to the second filtration [12, pages 403-4], which is defined as for the first filtration, but with the following definitions of $\check{O}, \check{R}$ and $\check{\leq}$ :

- $\check{a} \in$ Ǒ iff $(\exists a \in \check{a}) a \in O$,

- Rǎb̌c iff $(\exists a \in \check{a})(\exists b \in \check{b})(\exists c \in \check{c}) \operatorname{Rabc}$,

- $\check{a} \check{\leq} \check{b}$ iff $(\exists a \in \check{a})(\exists b \in \check{b}) a \leq b$.

However, as pointed out in [12, page 404], there is a problem with ensuring that the two bs in P2: $a \leq b \& R b c d \Rightarrow R a c d$, are the same after the definitions of $\check{a} \leq \check{b}$ and $\check{R} \check{b} \check{c} \check{d}$ are applied. We overcome this problem with the adoption of the simplified semantics, thus dropping ' $O$ ' and ' $\leq$ ' (except for logics containing AI2 but not R5). Moreover, there is a similar problem with the two postulates 'Rabz \& Rzcd $\Rightarrow(\exists x \in K)(R b c x \&$ Raxd)' and 'Rabz \& Rzcd $\Rightarrow(\exists x \in K)(\operatorname{Rac} x \& R b x d)$ ' in ensuring that the two $z$ are the same after the definitions of $\check{R}$ ǎb $\check{z}$ and $\check{R} \check{z} \check{c} \check{c}$ have been applied. So, we proceed to apply the second filtration to the F-logics, $\mathbf{B}^{\mathrm{d}}, \mathrm{DW}^{\mathrm{d}}, \mathrm{DJ}^{\mathrm{d}}, \mathrm{DK}^{\mathrm{d}}, \mathrm{DL}^{\mathrm{d}}$, $D^{d}, D C^{d}$ and $R B^{d}$, which do have simplified semantics and do not use these two postulates.

There is another similar problem for the postulate $\mathbf{P 2}$ of $\$ 2$, viz. RTab $\Leftrightarrow$ $a=b$, in that if $\check{R} \check{T} \check{a} \breve{b}$ is assumed then, for some $a, b \in K$, RTab may not hold for the specific base world $T$ in $\check{T}$. We overcome this by distinguishing in $\check{K}$ 
between $\check{T}$ and $\dot{T}$, where $\check{T}$ is $\{T\}$ and $\dot{T}$ is $\{b \in K: T=x b\}-\{T\}$. We also add $\check{T}^{*} *$ which is $\left\{T^{*}\right\}$, to be distinguished in $\check{K}$ from $\dot{T}^{*}$ which is $\left\{b \in K: T^{*}=x b\right\}-\left\{T^{*}\right\}$. [If $\dot{\mathrm{T}}$ is null, then delete $\dot{\mathrm{T}}$ and $\dot{\mathrm{T}}^{*}$ throughout the following argument.]

The base world in the second filtration is then taken to be $\check{T}$. We put $\check{T}$. as $\check{T}$, and $\dot{T}^{\check{*} *}$ as $\dot{T}$. We will continue to use á to represent elements of $\check{K}$ and $a \in \check{a}$ to represent elements of $K$ which are members of ă. For the definitions of $\check{R} \check{T} \breve{a} \breve{b}, \check{a} \check{\leq} \check{b}$ and $\check{v}(p, \check{a})$, we let the forms $a \in \check{a}$, etc. hold when $T \in \check{T}$ and $\mathrm{T} \in \check{\mathrm{T}}^{*}$, and for $\dot{\mathrm{T}}$ and $\dot{\mathrm{T}}^{*}$ in accordance with their set-theoretic memberships.

Lemma I Whenever $b \in \check{a}, b^{*} \in \check{a}^{*}$, for any element $\check{a}$ in $\check{K}$.

Proof For $\check{a}$, which is neither $\check{T}, \dot{T}, \check{T}^{*}$, nor $\dot{T}^{*}, a=x b$ and hence $a^{*}=x b^{*}$. Then $b^{*} \in a^{*}$ and, since $\check{a}^{*}={ }_{d f} a^{*}, b^{*} \in \check{a}^{*}$. If $b \in \check{T}$ then $b=T, b^{*}=T^{*}$ and $b^{*} \in \check{T}^{*}$. If $b \in \dot{T}$ then $b=x T$ and $b \neq T$. Hence $b^{*}=x T^{*}$ and $b \neq T^{*}$, and thus $b^{*} \in \dot{T}^{*}$. If $b \in \check{T}^{*}$ then $b=T^{*}, b^{*}=T, b^{*} \in \check{T}^{*}$ and $b^{*} \in \check{T}^{* * *}$. If $b \in \dot{T}^{*}$ then $b=x T^{*}$ and $b \neq T^{*}, b^{*}=x T$ and $b^{*} \neq T, b^{*} \in \dot{T}$ and $b^{*} \in \dot{T}^{* * *}$. $\#$

Theorem 5 Given a model $\mathfrak{M},\langle\mathrm{T}, \mathrm{K}, \mathrm{R}, *, \leq, v\rangle$, of the simplified semantics of one of the F-logics L, the second filtration X-model $\grave{\mathfrak{M}}$ : $\langle\grave{T}, \check{K}, \check{R}, \check{*}, \check{\leq}, \check{v}\rangle$, as defined above and with appropriate semantic postulates for $L$, is also a model of L. (' $\check{\leq}$ ' is included only for logics with AI2 but not R5.)

Proof We check the semantic postulates, PI-8, PII and PI2, listed in $\$ 2$ for the logics L. For the most part, [I2, pages 400-3], is followed.

PI. For ă, which is neither $\check{T}, \dot{T}, \check{T}^{*}$, nor $\dot{T}^{*}, \check{a}^{\check{*}}=\left(a^{* *}\right)^{\vee}=$ a. The cases for $\check{T}$, $\dot{\mathrm{T}}, \check{\top}^{*}$, and $\dot{\mathrm{T}}^{*}$ are clear from the above definitions.

P2. Let $\check{R} \check{T}$ ǎb. Then, by definition, RTab, for some $a \in \check{a}$ and $b \in \check{b}$, and hence $a=b$. Then $\check{a}=\check{b}$. Conversely, let $\check{a}=\check{b}$. Since RTaa, $\check{R} \check{T}$ ǎá and hence $\check{R} \check{T}$ ǎb̆.

P3. For ǎ, $\check{b}$ and $\check{c}$, which are neither $\check{T}, \dot{T}, \check{T}^{*}$, nor $\dot{T}^{*}$, let $\check{R}$ ǎb̌c. Then Rabc, for some $a \in \check{a}, b \in \check{b}, c \in \check{c}$, and hence $\operatorname{Rac}^{*} b^{*}$. Then $\check{R} \check{a}\left(c^{*}\right)^{\vee}\left(b^{*}\right)^{\vee}$ and $\check{R} \check{a} \check{c} \check{c}^{*} \check{b}^{*}$. By Lemma I, this result extends to the four additional cases.

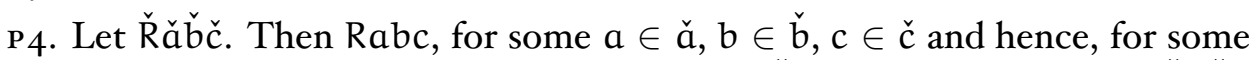
$x \in K, \operatorname{Rab} x$ and Raxc. Let $\check{x}$ be the member of $\check{K}$ such that $x \in \check{x}$. Then $\check{R} a \check{b} \check{x}$ and $\check{R}$ áx̌č for this $\check{x}$.

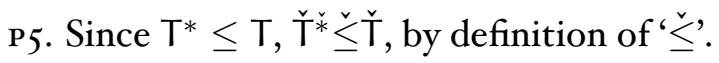

P6. For part (i), let $\check{a} \neq \check{T}$, then any such element $a$ of $K$, such that $a \in$ à, is not identical with T. Hence, for this a, Raa*a and, by Lemma I, Řáă $\check{a}^{*}$ ă. Part (ii) is clear from $\mathrm{P} 5$, when required.

P7. Similar to P4.

P8. Let $\check{a} \in \check{K}$. For any $a \in$ á, Raaa and hence Řǎǎă.

PII. Let Ǩ̌ ̌̌ ̌̌c. Then Rabc, for some $a \in \check{a}, b \in \check{b}, c \in \check{c}$, and hence Rbac and Rb̌ǎč. 
PI 2. We just consider $\check{T}=\check{T}^{*}$, in view of the logics L. Since $T=T^{*},\{T\}=\left\{T^{*}\right\}$ and $\check{T}=\check{T}^{*}$. For logics with AI 2 but not R5, we check the additional postulate, PI5, and the valuation condition VCI.

PI5. Since RT*aa, by definition, $R T^{*}$ áă.

vCI. Let $\check{v}\left(p, \check{T}^{*}\right)=\mathrm{T}$ and $\check{\mathrm{T}}^{*} \dot{\mathrm{C}} \check{\mathrm{T}}$. Then $v\left(\mathrm{p}, \mathrm{T}^{*}\right)=\mathrm{T}, \mathrm{p} \in \mathrm{X}$ and $\mathrm{T}^{*} \leq \mathrm{T}$. Hence $v(p, T)=T$ and $\check{v}(p, \check{T})=T$.

Let $\check{I}(A, \check{a})$ be the interpretation which extends the valuation $\check{v}(p, \check{a})$ to all formulae $A$ in accordance with the semantics.

Corollary I For logics with aI2 but not R5, with PI5 and vCI, for all formulae $A$, if $\check{I}\left(A, \check{T}^{*}\right)=T$ then $\check{I}(A, \check{T})=T$.

The following theorem shows that the second filtration interpretation $\check{I}(A, \check{a})$ over members of $X$ takes the same value as that of all of the interpretations $I(A, a)$, for $a \in \check{a}$.

Theorem 6 For the model $\mathfrak{M}$ and the X-model $\mathfrak{M}$ of Theorem 5, for $A \in X$, $\check{\mathrm{I}}(A, \check{a})=\mathrm{I}(A, a)$, for each $a \in$ à.

Proof We check this result inductively on formulae in $X$, for $a \in$ à. The argument is mostly as in [I2, page 4Or].

$p: \check{v}(p, \check{a})=T$ iff $v(p, a)=T$, for $p \in X$.

$\sim: \check{I}(\sim A, \check{a})=T$ iff $\check{I}\left(A, \check{a}^{*}\right)=F$, iff $\mathrm{I}\left(A, a^{*}\right)=F$, by the induction hypothesis, iff $\mathrm{I}(\sim A, a)=T$. We use Lemma I to ensure that $a^{*} \in \check{a}^{*}$ for the given $a \in$ á.

$\&, \vee$ : Straightforward.

$\rightarrow$ : Let $\check{\mathrm{I}}(\mathrm{A} \rightarrow \mathrm{B}, \check{a})=\mathrm{T}$. Then, for all $\check{b}, \check{c} \in \check{\mathrm{K}}$, if $\check{\mathrm{R} a ̌ b} \check{\mathrm{b}}$ and $\check{\mathrm{I}}(\mathrm{A}, \check{\mathrm{b}})=\mathrm{T}$ then $\check{I}(B, \check{c})=T$. Let Rabc and $I(A, b)=T$, for any $a \in \check{a}$ and $b, c \in K$. Then, by definition, $\check{R}$ á $\check{b} \check{c}$, where $b \in \check{b}, c \in \check{c}$, and, by induction hypothesis, $\check{\mathrm{I}}(\mathrm{A}, \breve{b})=\mathrm{T}$ and hence $\check{\mathrm{I}}(\mathrm{B}, \check{\mathrm{c}})=\mathrm{T}$ and $\mathrm{I}(\mathrm{B}, \mathrm{c})=\mathrm{T}$. So, $\mathrm{I}(\mathrm{A} \rightarrow \mathrm{B}, \mathrm{a})=\mathrm{T}$. Let $\check{I}(A \rightarrow B, \check{a})=F$. Then, for some $\check{b}, \check{c} \in \check{K}$, $\check{R}$ á $\check{b} \check{c}, \check{I}(A, \check{b})=T$ and $\check{\mathrm{I}}(\mathrm{B}, \check{\mathrm{c}})=\mathrm{F}$. By definition, Rabc, for some $a \in \check{a}, b \in \check{b}, c \in \check{c}$, and, by induction hypothesis, $I(A, b)=T$ and $I(B, c)=F$. Hence, $I(A \rightarrow B, a)=F$ for this $a \in$ à. However, since $A \rightarrow B \in X, I(A \rightarrow B, a)=F$ for all $a \in$ à. $\quad \sharp$ The corollary shows that the splitting of the original $X$-worlds $\check{T}$ and $\dot{T}$ does not affect their interpretations of formulae in $X$.

Corollary 2 For $A \in X, \check{I}(A, \check{T})=\check{I}(A, \dot{T})$ and $\check{I}\left(A, \check{T}^{*}\right)=\check{I}\left(A, \dot{T}^{*}\right)$, given that $\dot{\mathrm{T}}$ and $\dot{\mathrm{T}}^{*}$ are both non-empty and are thus proper elements of $\check{\mathrm{K}}$.

Proof By Theorem $6, \check{I}(A, \check{T})=I(A, T)$. There is an element $a \in \dot{T}$ such that $a=x T$ and $a \neq T$, and thus $I(A, T)=I(A, a)$, and, by Theorem 6 , $\check{I}(A, \dot{T})=I(A, a)$. So, $\check{I}(A, \check{T})=\check{I}(A, \dot{T}) . \check{I}\left(A, \check{T}^{*}\right)=\check{I}\left(A, \dot{T}^{*}\right)$ follows similarly, since, by Lemma $\mathrm{I}, \mathrm{a}^{*} \in \dot{\mathrm{T}}^{*}$. 
Theorem 7 Each F-logic ${ }^{\mathrm{d}}, \mathrm{DW}^{\mathrm{d}}, \mathrm{DJ}^{\mathrm{d}}, \mathrm{DK}^{\mathrm{d}}, \mathrm{DL}^{\mathrm{d}}, \mathrm{D}^{\mathrm{d}}, \mathrm{DC}^{\mathrm{d}}$ and $\mathrm{RBC} \mathrm{d}^{\mathrm{d}}$ is decidable.

Proof Each of the logics have the finite model property and are hence decidable. To show this, let $A$ be a non-theorem of one of the F-logics L. Then, by Theorems 2 and $4, A$ is invalid in the simplified semantics for $L$ and $I(A, T)=$ $F$, for some model $\mathfrak{M}$. Let $X$ be the appropriate closure of the formula $A$. By Theorems 5 and 6 , there is a second filtration $X$-model $\grave{\mathfrak{M}}$ such that $\check{I}(A, \check{T})=$ $F$, since $A \in X$. So, $A$ is falsified in the model $\check{\mathfrak{M}}$, which is finite since the set $\check{K}$ of $X$-worlds is finite. Decidability then follows [I2, pages 4OI-2].

The next theorem tightens this decidability argument further by showing that the theoremhood or non-theoremhood of a formula $A$ in an F-logic $L$ can be determined just by its $\mathrm{X}$-models.

Theorem 8 For a given formula $A, A$ is a theorem of an F-logic $L$ iff $A$ is true in all the $\mathrm{X}$-models of $\mathrm{L}$, where $\mathrm{X}$ is the appropriate closure of $\mathrm{A}$.

Proof If $A$ is a theorem of an F-logic $L$ then $A$ must be true in any of the $\mathrm{X}$-models, as they are models by Theorem 5 and soundness was shown by Theorems $\mathrm{I}$ and 3 . If $A$ is a non-theorem of $L$ then, by the argument in the proof of Theorem $7, \check{I}(A, \check{T})=F$ in an $X$-model $\check{\mathfrak{M}}$, where $X$ is the appropriate closure of the formula $A$.

The finite size of the $X$-models is determinable in terms of features of the formula $A$ under test. The size of $X$ is no more than twice the number of subformulae of $A$, taking into account the closure condition, and the number of $X$-worlds is limited to $2^{n}$, where $n$ is the number of formulae in $X$. In fact, starting with a formula $A$, we can identify each possible " $X$-world" by its set of interpretations ( $T$ or $F$ ) on the members of $X$, then choose $\check{T}$ and $\check{T}^{*}$ (and $\dot{T}$ and $\dot{\mathrm{T}}^{*}$ ) and define all the relation $\check{\mathrm{R}}$ and function $\check{*}$ (and also $\check{\leq}$, if necessary) on them to determine all the " $X$-models". These " $X$-models" and "X-worlds" within them are then pared down if semantic postulates or valuation or interpretation conditions fail, producing $X$-models that are appropriate for the F-logic L. Thus, all the possible $X$-models for $L$ can be constructed for the formula $A$, which, given Theorem 8 , can then be shown to be invalid, if $\check{I}(A, \check{T})=F$ for some $X$-model, or valid, if $\check{I}(A, \check{T})=T$ for all $X$-models. Though a decision procedure, this is very tedious and so we adapt this to the reductio method, commonly used in other areas of logic such as modal logic.

\section{The Ensuing Reductio Method}

In the Reductio Method, we start with the formula $A$ under test being assigned $F$ at $\check{T}$, and only construct what parts of the $X$-model(s) of the simplified semantics that are needed to try to falsify it. If an X-model can be determined, consistent with the semantic postulates and valuation and interpretation conditions of the F-logic L, $A$ is invalid and hence a non-theorem of L. If no such 
$\mathrm{X}$-models can be found, and this can only be registered in the derivation of contradictions in each of an exhaustive set of $X$-model attempts to falsify $A$, then $A$ is valid in all $X$-models and is hence a theorem of $L$.

We follow Hughes and Cresswell's reductio procedure for the modal logic т [9, pages 82-96] in setting out our reductio procedures for the F-logics L, but we need to include a rule for $*$-worlds and a rule for identifying worlds. We also need to distinguish between the two worlds $b$ and $c$ when Rabc holds, as they behave differently. We also need to add some further rules to ensure that the various semantic postulates for the logics L apply.

We set out the rules for the reductio procedure for our F-logics L, as follows: (Rules $\mathrm{I}-6$ are common to all logics L, whilst rule 7 is special to any of the particular logics L stronger than в.) (For simplicity, we drop the "'s,-notation.)

I. Put the formula $A$ under test in a rectangle, labelled with world $T$, and put the truth-value $F$ under its main connective.

We continue to use rectangles around members of $X$, generated from $A$ and taking truth-values $T$ or $F$ (put under the main connectives), and we append to each rectangle a world label. In fact, when we refer to worlds in the sequel we will assume an associated rectangle containing some members of $X$ with truth-values.

2. We apply the usual truth-table rules to the connectives \&, with $T$ under it, and $V$, with $F$ under it, to get definite values for the respective conjuncts and disjuncts. We continue to apply these truth-table rules to \& and $\vee$, where applicable, in any of the subsequent worlds.

3. For each un-starred world $a$, we introduce a $*$-world $a^{*}$ so that if a formula $\sim B$ takes $T(F)$ in world a then we put $B$ with value $F(T)$ in $a^{*}$, and if a formula $B$ takes $T(F)$ in world a and $\sim B \in X$ then we put $\sim B$ with $F(T)$ in $a^{*}$. Whether $a^{*}$ is a new world or not will depend on rule 5 and, in the case of T and $\mathrm{T}^{*}$, rule $7 \mathrm{D}$ or $7 \mathrm{H}$. (Note however that, for all logics $L, a^{* *}=a$, and that we constructed $a^{*}$ so that all the above assignments still apply when applied from $a^{*}$ into $a$, as well as applying from $a$ into $a^{*}$.)

4A. In a world $a$, if there is an ' $\rightarrow$ '-formula taking $F$ then there must be worlds $\mathrm{b}$ and $\mathrm{c}$, where we put the antecedent of the ' $\rightarrow$ ', with a $T$ under it, into the rectangle for $b$, and we put the consequent of the ' $\rightarrow$ ', with an $F$ under it, into the rectangle for $\mathrm{c}$. We pick a point on the outside of the rectangle for $\mathrm{a}$, draw arrows from this point to the rectangles for $\mathrm{b}$ and for $\mathrm{c}$, and draw a line (slash) through the stem of the arrow towards $b$, indicating that $b$ is to be treated differently from $c$ in relation to both this and the next clause. We call this an $R$-relation between $a, b$ and $c$, or just Rabc. We use a different starting point to draw such arrows for each ' $\rightarrow$ '-formula taking $F$ in $a$. Whether either or both of $b$ and $c$ are new worlds will depend on rule 5. (The rule does not have to be applied if the requisite worlds $b$ and $c$ already exist with the respective valuations.)

$4^{\text {B. }}$ In a world $a$, if there is an ' $\rightarrow$ '-formula taking $T$, then for each existing 
R-relationship between $a$ and worlds $b$ and $c$, shown by arrows from $a$ to $b$ with a slash and from $a$ to $c$ without a slash, both from the same point on $a$, if the antecedent of the ' $\rightarrow$ '-formula has a $T$ under it in the rectangle for $b$, then we put the consequent of the ' $\rightarrow$ ', with a $T$ under it, into the rectangle for $\mathrm{c}$.

4C. Since the postulate RTab $\Leftrightarrow a=b$ is common to all logics L, we introduce the single world a (with the two arrows from the T-rectangle to the a-rectangle) when evaluating an ' $\rightarrow$ '-formula, with an $F$ under it, in world $T$, putting both the antecedent and consequent of the ' $\rightarrow$ '-formula into a, with appropriate values. Whether $a$ is a new world will depend on rule 5. (The rule does not have to be applied if the requisite world a already exists with the respective valuations.) Further, for every world $b$, including $T, R T b b$ holds and so, when evaluating an ' $\rightarrow$ '-formula, with $T$ under it, we draw in the arrows so that whenever the antecedent of the ' $\rightarrow$ '-formula takes $T$ in $b$ the consequent takes $\mathrm{T}$ in $\mathrm{b}$.

5C. Any two worlds with the same value assignments for all the members of $X$ must be identified. This means that the same rectangles are used for both these worlds and thus the same $*$ - and R-relationships hold for these worlds. However, there are exceptions for T and T*. All the worlds having the same values over $X$ as for $T$ are put identical to $\dot{T}$, and all the worlds having the same values over $\mathrm{X}$ as for $\mathrm{T}^{*}$ are put identical to $\dot{T}^{*}$. Moreover, one can get to situations where there are no further distinct $\mathrm{X}$-worlds and in the application of rule $4 \mathrm{~A}$ or even rule 3 one is left to consider all the possible identifications between the worlds needed for these rules and the existing worlds.

5в. For the purpose of trying to establish a consistent $X$-model, we may choose to make an identification between worlds so that there is a consistent assignment of values to the formulae in the identified world. If this indeed yields an X-model, this will result in a restricted form of the X-model one is working with. We will call this a restricted $X$-model. If a contradiction in another world is derived from this identification then this contradiction only applies to this particular identification and other identifications would have to be tried to see whether $\mathrm{X}$-models or contradictions result.

6. In applying the usual truth-table rules to the connectives \&, with $F$ under it, and $V$, with $T$ under it, we get three different value combinations for the respective conjuncts and disjuncts. These yield what we call alternative $\mathrm{X}$-models. Whilst any of these alternatives would suffice as an X-model, contradictions would need to be established in all three to produce a general contradiction for the procedure at this point.

Rules I-6 apply for the logic в. For stronger logics L, we now add special rules applicable for the particular logic $L$ we are dealing with. We examine the various semantic postulates of $\$ 2$ in turn. Note that different starting points are used for each pair of arrows representing an R-relation, drawn from any single rectangle. 
7A. For P3. Rabc $\Rightarrow R a c^{*} b^{*}$, we add the R-relation $R a c^{*} b^{*}$ to each existing $R$-relation Rabc, inserting formulae and values according to rule $4^{\mathrm{B}}$. We do not need to re-apply this rule to $\operatorname{Rac}^{*} b^{*}$ as $\operatorname{Rab}^{* *} c^{* *}$ already holds.

7в. For $\mathbf{P} 4$. Rabc $\Rightarrow x \in K, R a b x$ and Raxc, we add a world $x$ and the Rrelations Rabx and Raxc to each existing R-relation Rabc. We insert formulae and values according to rule $4 \mathrm{~B}$ for both Rabx and Raxc. (The rule does not have to be applied if the requisite world $x$ already exists with the respective R-relations.)

Realising that this process could run on until all $X$-worlds are exhausted, I would suggest identifying $x$ with $b$, if possible, in accordance with rule 5 в. For then 7 в need not be re-applied to Rabb.

7C. For $\mathrm{P} 5 . \mathrm{T}^{*} \leq \mathrm{T}$, we introduce the special $\leq$-relationship, indicated by a single arrow from $T^{*}$ to $T$, in which every formula taking $T$ at world $T^{*}$ is given the value $T$ in world $T$ and every formula taking $F$ at $T^{*}$ is given the value $F$ at $T$.

7D. For $\mathrm{p} 6$. Raa*a, we add this R-relation for each world a other than $T$ and apply rule $4^{\mathrm{B}}$. $\mathrm{T}^{*} \leq \mathrm{T}$ is then added as for rule $7 \mathrm{C}$. If $\mathrm{T}=\mathrm{T}^{*}$ applies, then identify these worlds as well. By rule $5 \mathrm{~A}$, this will imply the identification of $\dot{T}$ and $\dot{\mathrm{T}}^{*}$, as formulae would have the same values in $\dot{\mathrm{T}}^{*}$ and $\dot{\mathrm{T}}^{*}$.

7E. For $\mathrm{P} 7$. Rabc $\Rightarrow(\exists x \in \mathrm{K}) \operatorname{Rabx} \& \mathrm{R} x \mathrm{bc}$, we proceed in a similar manner to rule $7 \mathrm{~B}$, but I suggest identifying $x$ with $\mathrm{a}$, for then $7 \mathrm{E}$ need not be re-applied to Raba.

7F. For $\mathrm{P} 8$, Raaa, we add this R-relation for each world $\mathrm{a}$ and apply rule $4^{\mathrm{B}}$.

7G. For PII. Rabc $\Rightarrow$ Rbac, we proceed in a similar manner to rule $7 \mathrm{~A}$, and we do not need to re-apply this rule to Rbac.

$7 \mathrm{H}$. For PI2. $\mathrm{T}=\mathrm{T}^{*}$, we identify these worlds. As for rule $7 \mathrm{D}, \dot{\mathrm{T}}$ and $\dot{\mathrm{T}}^{*}$ are also identified. (We note that $\mathrm{T} \leq \mathrm{T}^{*}$ does not arise for the logics L.)

The following theorem states the relationship between the reductio method and theoremhood for the F-logics L.

Theorem 9 A formula $A$ is a theorem of an F-logic $L$ if a contradiction is obtained in each of an exhaustive set of derived $X$-models for $L$ that assign the value $F$ to $A$ at world $\check{T}$. $A$ is a non-theorem of the logic $L$ if a consistent $X$-model for $L$ is obtained which assigns the value $F$ to $A$ at world $\check{T}$.

Proof Clearly, if a contradiction is obtained in each of an exhaustive set of derived $X$-models for $L$ that assign the value $F$ to $A$ at world $\check{T}$ then there are no consistent such $X$-models for $A$ and $\check{I}(A, \check{T})=T$ for all $X$-models of $L$. Then, by Theorem $8, A$ is a theorem of $L$. In the process of exhausting a set of derived $X$-models for $L$ one must include all alternative $X$-models under rule 6 and all restricted $X$-models under rules $5 \mathrm{~A}$ and $5 \mathrm{~B}$. On the other hand, if a consistent $X$-model for $L$ is obtained which assigns the value $F$ to $A$ at world $\check{T}$ then $\check{I}(A, \check{T})=F$ for this $X$-model and, by Theorem $8, A$ is a non-theorem of $L$. 


\section{The Reductio Method for Contraction-less Logics}

We proceed to develop a similar reductio method for the contraction-less logics $B^{\mathrm{d}}, \mathrm{DW}^{\mathrm{d}}, \mathrm{TW}^{\mathrm{d}}$ and $\mathrm{RW}{ }^{\mathrm{d}}$, and, except for $\mathrm{RW}^{\mathrm{d}}$, systems obtained by the addition of AI2. $A \vee A$ (add ' $x$ ' to name, prior to 'd') and systems obtained by the addition of both AI2 and R5. $\sim A, A \vee B \Rightarrow B$ (add ' $C$ ' to name, prior to 'd'). These are called the $\mathrm{u}$-logics in $\$ 2$. As indicated in $\$ 3$, the first and second filtrations will not work for the (new) axioms AI 6 and AI 7 of $\mathrm{TW}$, and so, we need another approach. (Recall that $\mathbf{B}=\mathbf{B}^{\mathrm{d}}$, $\mathrm{DW}=\mathrm{DW}^{\mathrm{d}}$, $\mathrm{TW}=\mathrm{TW}^{\mathrm{d}}$ and $\mathrm{RW}=\mathrm{RW}^{\mathrm{d}}$. We will drop the 'd, when naming these systems for simplicity.)

We will not use a filtration method and tackle the semantics directly. Since B and Dw have already been treated in $\$ 4$, we start by focussing on Tw. If we consider the two semantic postulates for AI 6 and AI7 of TW, viz.

P9 $\operatorname{Rabz} \& \operatorname{Rzcd} \Rightarrow \exists x \in \mathrm{K}$, Racx \& Rbxd, and

PIo $\operatorname{Rab} z \& \operatorname{Rzcd} \Rightarrow \exists x \in \mathrm{K}, \mathrm{Rbcx} \& \operatorname{Raxd}$

there seems to be a limit to the number of re-applications of these postulates if the introduced worlds $x$ are new on each occasion and there are no postulates, further than those for $\mathrm{Tw}$, to concern us, at least for the moment. In order to apply these postulates there must be two R-relations of the form Rabz and Rzcd, and the introduced $x$ cannot play the role of the $z$ as they stand, which means that we have to look around at our original set of R-relations for other worlds for $z$ to equal. This means that $z$ must ultimately come from the finite set of worlds and R-relations that we would already have from the reductio method to this point and, if we insist that the introduced worlds are all new, these two postulates will not have the propensity to generate an infinite number of worlds. We set this argument out more fully below.

We will continue to use the simplified semantics as it will give a simpler and more straight-forward decision procedure. Assuming the introduction of such new worlds for the logic Tw, we set out the rules for the reductio procedure, much as in $\$ 4$ :

I. Put the formula $A$ under test in a rectangle, labelled with world $T$, and put the truth-value $F$ under its main connective.

2. We apply the usual truth-table rules, in this and subsequent worlds, to the connectives \&, with $\mathrm{T}$ under it, and $\mathrm{V}$, with $\mathrm{F}$ under it.

3. For each un-starred world $a$, we introduce a $*$-world $a^{*}$ so that if a formula $\sim B$ takes $T(F)$ in world $a$ then we put $B$ with value $F(T)$ in $a^{*}$, and if a formula $B$ takes $T(F)$ in world $a$ then we put $\sim B$ with $F(T)$ in $a^{*}$. $a^{*}$ is to be a new world, unless there is already a world with all these properties. (Note as before that $a^{* *}=a$.)

4A. In a world $a$, if there is an ' $\rightarrow$ '-formula taking $F$ then there must be worlds $\mathrm{b}$ and $\mathrm{c}$, where we put the antecedent of the ' $\rightarrow$ '-formula, with a $\mathrm{T}$ under it, 
into the rectangle for $b$, and we put the consequent of the ' $\rightarrow$ ', with an $F$ under it, into the rectangle for $\mathrm{c}$. As before, we draw arrows from a point on $a$ to the rectangles for $b$ and for $c$, with a slash through the arrow towards $b$. We call this an R-relation between $a, b$ and $c$, or just Rabc. $b$ and $c$ are both taken to be new worlds, except as for rule $4 \mathrm{C}$ below. These worlds are new with respect to existing worlds and their $*$-worlds. (The rule is not applied if the requisite worlds $b$ and $c$ already exist with the respective valuations.)

4B. In a world $a$, if there is an ' $\rightarrow$ '-formula taking $T$ then, for each existing R-relationship between $a$ and worlds $b$ and $c$, shown by arrows from $a$ to $b$ with a slash and from $a$ to $c$ without a slash, both from the same point on $a$, if the antecedent of the ' $\rightarrow$ '-formula has a $T$ under it in the rectangle for $b$, then we put the consequent of the ' $\rightarrow$ ', with a T under it, into the rectangle for $\mathrm{c}$.

4C. Since, for the simplified semantics, RTab $\Leftrightarrow a=b$, we introduce the single world $a$ (with the two arrows from the T-rectangle to the a-rectangle) when evaluating an ' $\rightarrow$ '-formula, with an $F$ under it, in world $T$, putting both the antecedent and consequent of the ' $\rightarrow$ '-formula into $a$, with appropriate values. Similarly to $4 \mathrm{~A}$, the world $\mathrm{a}$ is new, unless such a world already exists. Further, for every world $b$, including $T, R T b b$ holds and so, when evaluating an ' $\rightarrow$ '-formula, with $T$ under it, we draw in the arrows so that whenever the antecedent of the ' $\rightarrow$ '-formula takes $T$ in $b$ the consequent takes $T$ in $b$.

6. In applying the usual truth-table rules to the connectives \&, with $F$ under it, and $\vee$, with $T$ under it, we get three different value combinations for the respective conjuncts and disjuncts. These yield what we call alternative models.

7A. We add the R-relation Rac*b* to each existing R-relation Rabc, inserting formulae and values according to rule $4 \mathrm{~B}$.

7B. For each existing pair of R-relations Rabz and Rzcd, we add the R-relations Racx and Rbxd, and Rbcy and Rayd, where both worlds $x$ and $y$ are new (i. e. new to existing worlds and their $*$-worlds), inserting formulae and values according to rule $4 \mathrm{~B}$. This rule is not applied when such $x$ or $y$ already exist with the requisite R-relations.

For the purposes of comparing this reductio procedure with one based precisely on the simplified semantics for $\mathrm{Tw}$, we consider, in the proof below, the addition of the following rule:

5. We can identify worlds $a^{*}$, introduced in rule 3 , with any existing world, as we can for the worlds $\mathrm{b}$ and $\mathrm{c}$, introduced in rule $4 \mathrm{~A}$, the world $\mathrm{a}$, introduced in rule $4 \mathrm{C}$, and also the worlds $x$ and $y$, introduced in rule $7 \mathrm{~B}$, whether such an existing world initially had the requisite properties or not.

We now state the relationship between the above reductio method and theoremhood for $\mathrm{Tw}$, the proof of which involves a general argument concerning the use of new worlds. 
Theorem io A formula $A$ is a theorem of Tw if a contradiction is obtained in each of an exhaustive set of derived models that assign the value $F$ to $A$ at world T. A is a non-theorem of $\mathrm{Tw}$ if a consistent model is obtained which assigns the value $F$ to $A$ at world $T$.

Proof If a consistent model is obtained which assigns the value $F$ to $A$ at world $T$ then $I(A, T)=F$ for this model and $A$ is invalid in the simplified semantics for Tw. By Theorem 3 of $\$ 2, A$ is a non-theorem of $T w$. (Note that $\mathrm{TW}=\mathrm{TW}^{\mathrm{d}}$.)

Let a contradiction be obtained in each of an exhaustive set of derived models that assign the value $F$ to $A$ at world $T$. These contradictions would exhaust a set of alternative models, but would be based on the above rules 3 , $4^{\mathrm{A}}, 4^{\mathrm{C}}$ and $7 \mathrm{~B}$, where new worlds were chosen for $\mathrm{a}^{*}, \mathrm{~b}, \mathrm{c}, \mathrm{a}, \mathrm{x}$ and $\mathrm{y}$. If we were to add rule 5 and allow identifications of these worlds with pre-existing worlds then the argument to the contradiction(s) would still apply as there would be no changes to the existing assignments of values in the now identified worlds. Indeed, the identifications may induce further contradictions. There may also be further applications of rule 7 в due to the identifications, but this is not going to remove any derived contradictions. Also, any contradiction based on the lack of application of the rules $3,4 \mathrm{~A}, 4 \mathrm{C}$ and $7 \mathrm{~B}$, due to the presence of pre-existing worlds with the requisite properties, would still apply if such rules were nevertheless applied to introduce new worlds or other worlds in conjunction with rule 5 . Thus, we have established the contradiction(s) within the simplified semantics for $\mathrm{Tw}$, and hence there can be no consistent models for $A$ such that $I(A, T)=F$. So, $I(A, T)=T$ for all models and $A$ is valid in the simplified semantics for Tw. Hence, by Theorem 4 of $\$ 2, A$ is a theorem of $\mathrm{TW}$.

We now proceed with the decidability argument based on the reductio method for Tw.

Theorem I I TW is decidable, using the above reductio method.

Proof The reductio method for Tw builds up a set of worlds, starting with $\mathrm{T}$, existentially introducing new worlds using the rules $3,4 \mathrm{~A}, 4 \mathrm{C}$ and $7 \mathrm{~B}$, the latter three with new R-relations. We also introduce new R-relations using the rules $4 \mathrm{~B}, 4 \mathrm{C}$ and $7 \mathrm{~A}$. We examine the various types of world and R-relation introduction. Rules 3 and most of 4 are formula-based, in that the presence of a formula induces the rule, whilst rule 7 and the second part of $4 \mathrm{C}(4 \mathrm{C}-2)$ involving the R-relation RTbb are not, in that the rule applies to any existing world or when an R-relation or certain configuration of R-relations occurs. Rules 3 and $7 \mathrm{~A}$ are idempotent, i. e. the original world or R-relation is reached after two applications of the respective rule. The rule $4 \mathrm{C}-2$ applies only to the pre-existing worlds $b$.

So, the rules 3, 4A-C and $7 \mathrm{~A}$ are either formula-based or idempotent or rely on pre-existing worlds. (We also use this terminology for the associated 
R-relations.) A reductio system, all of whose rules are of one of these three types, would be decidable as only finitely many worlds could be generated, the number of which would be dependent upon the number of subformulae in the formula under test, and so the number of generated R-relations would also be finite.

The only rule that is likely to cause trouble as far as decidability is concerned is rule $7 \mathrm{~B}$, as it may be capable of generating, through successive applications, an infinite sequence of worlds. We examine the possibilities for this.

First consider the interaction of rule $4 \mathrm{C}-2$, which yields RTaa. Let this supply one of the two R-relations, Rabz and Rzcd. If RTaa and Racd then RTcx and Raxd, for some $x$, and Racy and RTyd, for some $y$. We do not apply rule $7 \mathrm{~B}$ here as can be seen by putting $x=\mathrm{c}$ and $y=d$. Let us consider RabT and RTcc. The only way RabT can be introduced is by rule $4 \mathrm{C}-2$, in which case, $a=b=T$ and this becomes an instance of the first case. Clearly, RabT is not formula-based. The problem with introducing RabT using rule $7 \mathrm{~B}$ with ' $\mathrm{T}$ ' in the d-position is that ultimately one must start with RTTT and RTTT, which is also an instance of the first case above. If we try to derive $\operatorname{RaT}^{*} \mathrm{~b}^{*}$ using rule $7 \mathrm{~B}$, one must start with something of the same form, which is again not formula-based. As a result we can assume that both the Rabz and the Rzcd are generated by rule 4 and/or rule 7 в. Rule 7 A does not have a significant impact due to its idempotence. Consider an application of rule $7 \mathrm{~B}$ in the forms, Rabz and Rzcd $\Rightarrow$ Racx and Rbxd, for some world $x$; and Rabz and Rzcd $\Rightarrow$ Rbcy and Rayd, for some world $y$. Since $x$ and $y$ are new, there is no immediate generation of further worlds by application of rule 7 в. Also, the R-relations that start such applications are all formula-based and so are finite in number. Since each world introduced by rule 4 is new, we would have finite strings of these R-relations all linked together like Rabz and Rzcd are, or like Rabz and Rbcd, depending on the shape of the formulae involved. Other than the link $z$, the other worlds are all different, and when rule 7 в is applied this link is replaced by other linking worlds $x$ and $y$.

We consider a string of linked formula-based R-relations of length $n$, linked so that rule $7 \mathrm{~B}$ can apply between successive R-relations. We assume that a finite number of worlds are generated by an exhaustive set of applications of rule 7 в to this string. We then add another linked formula-based R-relation, Rdef, to the end of the string, making it a string of length $n+1$, and show that there are only finitely many extra worlds introduced by exhaustive applications of rule 7 в.

Consider R-relations of the type Rayd and Rbxd, obtained after any (finite) number of applications of $7 \mathrm{~B}$ to the initial string of length $n$, where the ' $x$ ' and ' $y$ ' are worlds introduced by the last such application of 7 в and ' $a$ ' and ' $b$ ' can either be worlds from a formula-based R-relation Rabz or introduced bу 7 в like the ' $x$ ' or the ' $y$ ' worlds but earlier. The R-relations Rayd and Rbxd are the only types that can interact with Rdef using rule 7 в. So, in applying 7 в here, we obtain Raex' and Ryx'f, Ryey' and Ray'f, Rbex" and Rxx"f, and Rxey" 
and Rby"f, where $x^{\prime}, y^{\prime}, x^{\prime \prime}$ and $y^{\prime \prime}$ are all new. To take this further, each of the worlds $a, b, x$ and $y$ in the first positions may link up with an R-relation with these worlds in third position, such an R-relation being obtained from a number of applications of 7 в to the initial string of length $n$. Such worlds $a$, $b, x$ and $y$ would have been introduced by an application of $7 \mathrm{~B}$, involving for example something of form Rx̀ $c x$ for the ' $x$ ', where ' $x$ ' and ' $c$ ' are worlds from a formula-based R-relation or introduced earlier (than ' $x$ ') by 7 в. Applying 7в to $R \grave{x} c x$ and Rxey" yields Rx̀ex"' and Rcx ${ }^{\prime \prime \prime} y^{\prime \prime}$, and Rcey "' and Rx̀y $y^{\prime \prime \prime} y^{\prime \prime}$, for new $x^{\prime \prime \prime}$ and $y^{\prime \prime \prime}$. There may be other subsequent occurrences of such forms Rìcx obtained by subsequent applications of 7 в, but the same structure applies. One can again take this further by linking up with the worlds $\grave{x}$ and $c$. What is apparent however is that on each such linkage the worlds are introduced earlier and earlier until, by successive application, there is no such linkage at all due to the worlds being part of a formula-based R-relation. So the whole process is finite, introducing only finitely many new worlds and R-relations. Note that there is no generation of further worlds by linking any of the newly introduced worlds $x^{\prime}, y^{\prime}, x^{\prime \prime}, y^{\prime \prime}, x^{\prime \prime \prime}, y^{\prime \prime \prime}$, etc. as they cannot appear in first position without an R-relation of form Rfgh. Also note that application of $7 \mathrm{~A}$ would just give a $*$-version of this whole process.

So, by an induction argument, only finitely many worlds can be introduced by rule 7 в from any string of linked formula-based R-relations of any finite length. This addresses our only remaining concern and thus $\mathrm{Tw}$ is decidable, using the reductio method.

Corollary 3 в and Dw are decidable.

Proof The reductio method for Dw is obtained by removing rule 7 в from that for $\mathrm{TW}$ and the reductio method for $\mathrm{B}$ is obtained by removing rule $7 \mathrm{~A}$ from that for Dw. Hence Dw and $\mathrm{B}$ are both decidable by these methods as, with the absence of $7 \mathrm{~B}$, there is no impediment to the finiteness of the set of introduced worlds, as mentioned in the above proof.

We now extend the decidability argument for $\mathrm{Tw}$ to $\mathrm{RW}$ and then to the remaining U-logics.

TheOREM I2 RW is decidable.

Proof We first simplify the formulae that we need to consider so that the proof can go through. As shown by Slaney [13], it suffices to consider just entailments of form $A \rightarrow B$ when determining theorems of $\mathrm{Rw}$, due to the primeness of theorems, viz. $\vdash A \vee B$ iff $\vdash A$ or $\vdash B$, and the break up of negated entailments, viz. $\vdash \sim(A \rightarrow B)$ iff $\vdash A$ and $\vdash \sim B$. So, for the reductio method, we do not have to consider negated entailments being true at world $T$ nor entailments false at $\mathrm{T}^{*}$, and so the formula-based R-relation $\mathrm{RT}^{*} \mathrm{ab}$, and its equivalents $\mathrm{RaT}^{*} \mathrm{~b}$ and $\mathrm{Rab}^{*} \mathrm{~T}$ (see rule $7 \mathrm{C}$ below), would never arise. However, $\mathrm{RT}^{*} \mathrm{TT}^{*}$ can be derived, as can be seen from rule $7 \mathrm{C}$ below. 
With the addition of the semantic postulate PII. Rabc $\Rightarrow$ Rbac, we add the following rule to those in the reductio method for $\mathrm{Tw}$ :

7C. We add the R-relation Rbac to each existing R-relation Rabc, inserting formulae and values according to rule $4 \mathrm{~B}$.

As for rule $7 \mathrm{~A}$, this rule is idempotent. However, unlike $7 \mathrm{~A}$ which is fairly harmless, rule $7 \mathrm{C}$ leads to some complications which will need to be examined. In conjunction with $7 \mathrm{C}, 7 \mathrm{~B}$ will allow the introduced worlds $x$ and $y$ to be positioned in such a way as to allow 7 в to be re-applied with $x$ and $y$ as links. Also, given 4C, rule $7 \mathrm{C}$ will yield RaTa, which with 7 в will generate an infinite number of worlds, as we will shortly see for $\mathrm{Ew}^{\mathrm{d}}$. However, in this case, $7 \mathrm{C}$ will prevent that from happening. Further, using $7 \mathrm{~A}$ and $7 \mathrm{C}$, we get $\mathrm{Raa}^{*} \mathrm{~T}^{*}$, which also needs examination.

What we will show here is that there are no more new worlds introduced by the addition of rule $7 \mathrm{C}$. New worlds can only be generated in conjunction with 7B where, given Rabz and Rzcd, we add Racx and Rbxd, for some world $x$, and Rbcy and Rayd, for some world $y$. We assume that $7 \mathrm{C}$ is applied before $7 \mathrm{~B}$, wherever possible, as a matter of procedure. We address the three abovementioned concerns in turn.

(i) We expand the conclusions of $7 \mathrm{~B}$ by using $7 \mathrm{C}$, as follows: Racx and Rxbd, Rcax and Rxbd, Rbcy and Ryad, Rcby and Ryad. To each of these forms, we can re-apply 7в, as follows: Rabz and Rczd, Rcby and Rayd, Rcby and Rayd, $R a b z$ and Rczd, Rbaz and Rczd, Rcax and Rbxd, Rcax and Rbxd, Rbaz and Rczd. We fill in the variable places in accordance with existing R-relations, taking into account applications of $7 \mathrm{C}$ in the process. So, any re-application of $7 \mathrm{~B}$, as a result of $7 \mathrm{C}$, does not yield any new worlds. Also note that application of $7 \mathrm{~A}$ would just give equivalent $*$-versions of this process.

(ii) We consider the use of RaTa with 7 в. Similarly to the case where RTaa was in the Rabz position, from RaTa and Rabc we get Racx and RTxd, and RTcy and Rayd, where by putting $x=d$ and $y=c$ we obtain pre-existing R-relations. Also, when we consider Rabc and RcTc, we get RaTx and Rbxc, and $b T y$ and Rayc. Here, we put $x=a$ and $y=b$ to obtain pre-existing R-relations, with help from $7 \mathrm{C}$.

(iii) In considering $\operatorname{Raa}^{*} \mathrm{~T}^{*}$ with $7 \mathrm{~b}$, from $R a b c$ and $\operatorname{Rcc}^{*} \mathrm{~T}^{*}$ we get $\operatorname{Rac}^{*} \chi$ and $\mathrm{RbxT}^{*}$, and $\mathrm{Rbc}^{*} y$ and $R a y T^{*}$, where by putting $x=b^{*}$ and $y=a^{*}$ we obtain pre-existing R-relations. However, with $\mathrm{Raa}^{*} \mathrm{~T}^{*}$ in the Rabz position, we would need an R-relation of the form $R T^{*} c d$, which is not formula-based, as seen above. Unless $c=T$ and $d=T^{*}, R T^{*} c d$ is not generated by using $7 \mathrm{~B}$, since ultimately one of the forms $\mathrm{RT}^{*} \mathrm{~cd}, \mathrm{RcT}^{*} \mathrm{~d}$ or RcdT would need to be formula-based. If we apply 7 в to $\mathrm{Raa}^{*} \mathrm{~T}^{*}$ and $\mathrm{RT}^{*} \mathrm{TT}^{*}$ we get $\mathrm{RaT} x$ and $\mathrm{Ra}^{*} \chi \mathrm{T}^{*}$, and $R^{*} T y$ and RayT*, where we can put $x=a$ and $y=a^{*}$.

There are no other ways that new worlds might be introduced by $7 \mathrm{C}$ that would 
not be introduced otherwise, and so $\mathrm{RW}$ is decidable, given the argument for Tw.

Theorem i3 All the U-logics are decidable.

Proof We just need to examine the addition of AI2. $A \vee \sim A$ and of AI2 together with R5. $\sim A, A \vee B \Rightarrow B$, to B, DW and Tw. For AI2, we add the semantic postulate $\mathrm{P}_{5}$, together with the ordering relation ' $\leq$ ', the valuation condition VCI and PI5. RT*aa. We add the following rule $7 \mathrm{D}$ to the reductio method.

$7 \mathrm{D}$. We introduce the special $\leq$-relationship, indicated by a single arrow from $T^{*}$ to $T$, in which every formula taking $T$ at world $T^{*}$ is put with value $T$ in world $T$ and every formula taking $F$ at $T^{*}$ is put with value $F$ at $T$. We also add the R-relation $\mathrm{RT}^{*} \mathrm{aa}$, for all existing worlds a.

For $\mathrm{BX}^{\mathrm{d}}$ and $\mathrm{Dw} \mathrm{x}^{\mathrm{d}}, 7 \mathrm{D}$ does not change the finitude of the worlds. For $\mathrm{Tw}^{\mathrm{d}}{ }^{\mathrm{d}}$, we need to examine the effect of $\mathrm{RT}^{*}$ aa on $7 \mathrm{~b}$. Firstly, $\mathrm{RT}^{*} \mathrm{aa}$ and Rabc yield $R T^{*} b x$ and Raxc, and Raby and $R T^{*} y c$, where we can put $x=b$ and $y=c$. Secondly, consider RabT* and $R^{*} c c$, whereupon RabT* (and RaTb*) is not formula-based and cannot be obtained through applications of $7 \mathrm{~B}$, unless it is one of RTT* $T^{*}$ or $R T^{*} T^{*} T^{*}$. The first of these is covered by RTaa and the second by RT*aa above.

For $\mathrm{R}_{5}$, we add $\mathrm{PI}$. $\mathrm{T} \leq \mathrm{T}^{*}$, which becomes $\mathrm{T}=\mathrm{T}^{*}$ in the presence of $\mathrm{P} 5$. We modify rule 3 and add rule $7 \mathrm{E}$ below.

3. $a^{*}$ is a new world for $a \neq T$.

$7 \mathrm{E}$. We put $\mathrm{T}^{*}$ identical with $\mathrm{T}$.

For $\mathrm{BC}^{\mathrm{d}}$ and $\mathrm{DWC}^{\mathrm{d}}$ and $\mathrm{TWC}^{\mathrm{d}}$, there is little change from their respective systems, $\mathrm{B}^{\mathrm{d}}$, Dw ${ }^{\mathrm{d}}$ and $\mathrm{Tw}^{\mathrm{d}}$.

We next examine some of the major extensions of the U-logics to see what goes wrong with the above decidability arguments and why they might thus be undecidable, though this argument itself will not constitute a proof as it deals with only one method, viz. this particular reductio method. The logics R, E and $\mathrm{T}$ have already been proved to be undecidable by Urquhart [ 15$]$. In fact, he proved undecidability for all logics between TW+AI5 and R [15, pages I069-70]. Perhaps his methods can be extended to other systems where undecidability is suggested in this paper but not already shown.

We start with $\mathrm{Ew}^{\mathrm{d}}$, which is $\mathrm{Tw}^{\mathrm{d}}+\mathrm{R} 6$, where $\mathrm{R} 6$ is the rule, $A \Rightarrow A \rightarrow \mathrm{B} \rightarrow$ $B$. Using simplified semantics, its corresponding semantic postulate is PI7: $\mathrm{RaTa}$, which in the absence of $7 \mathrm{C}$ does lead to the following infinite sequence of worlds. Let Rabc. Then, since RcTc, by applying 7в, we get RaTx and $\mathrm{Rbxc}$, for some new $x$. Then consider Rbxc and RcTc, in which case, RbTx' and $R x x^{\prime} c$, for some $x^{\prime}$. Further, $R x x^{\prime} c$ and $R c T c$ yields $R x T x^{\prime \prime}$ and $R x^{\prime} x^{\prime \prime} c$, for some $x^{\prime \prime}$. Thus, an infinite sequence of worlds can be generated. 
We next consider $\mathrm{Rw} \mathrm{X}^{\mathrm{d}}$ and $\mathrm{RWC}^{\mathrm{d}}$, obtained from $\mathrm{RW}^{\mathrm{d}}$ by the successive addition of $\mathrm{AI} 2$ and $\mathrm{R} 5$. With the addition of $\mathrm{AI} 2$, we add the rule $7 \mathrm{D}$, which includes the R-relation $\mathrm{RT}^{*} \mathrm{bb}$, for any $\mathrm{b}$. However, we can combine this with $R_{a a^{*}} T^{*}$ using 7 в to get Rabx and $\operatorname{Ra}^{*} x b$, for some $x$, and $R a^{*}$ by and Rayb, for some $y$. Using these new worlds $x$ and $y, R x x^{*} T^{*}$ and $R T^{*} y y$, and hence Rxy $x^{\prime}$ and $R x^{*} x^{\prime} y$, and $R x^{*} y y^{\prime}$ and $R x y^{\prime} y$, for new $x^{\prime}$ and $y^{\prime}$. We can thus generate an infinite sequence of worlds employing the rules $4 \mathrm{C}, 7 \mathrm{~A}, 7 \mathrm{C}$ and $7 \mathrm{D}$ as they stand. For RwC ${ }^{d}$, we similarly use Raa*T and RTbb.

We next consider any extension of $\mathrm{TW}^{\mathrm{d}}, \mathrm{EW}^{\mathrm{d}}$ or $\mathrm{RW}^{\mathrm{d}}$ consisting of one or more of the following axioms: AII, AI3, AI4, AI5, with corresponding semantic postulates: P4, P6, P7, P8. (However, for any such system $L$ without $\mathrm{R} 6$ and either without AI2 or with $\mathrm{AI}_{5}, \mathrm{~L}^{\mathrm{d}}=\mathrm{L}$.) These systems include $\mathrm{TJ}, \mathrm{T}, \mathrm{E}$ and $\mathrm{R}$, where $\mathrm{TJ}=\mathrm{TW}+\mathrm{AII}, \mathrm{T}=\mathrm{TW}+\mathrm{AI} 3+\mathrm{AI} 4, \mathrm{E}=\mathrm{EW}+\mathrm{AI} 3+\mathrm{AI} 4, \mathrm{R}=$ $\mathrm{RW}+\mathrm{AI} 4 . \mathrm{P} 4$ and $\mathrm{P} 7$ clearly lead to an infinite sequence of worlds by successive re-application. $\mathrm{P} 6$ and $\mathrm{P} 8$ are both of the form Raba, for some $\mathrm{b}$. This form generally leads to an infinite progression by applying 7 в as follows. Raba and Racd yields Racx and Rbxd, and Rbcy and Rayd. In turn, Raba and Racx yields Racx and $R b x^{\prime} x$, and Raba and Racx' yields Racx" and $R b x^{\prime \prime} x^{\prime}$. The avoidance of the form Raba is one of the reasons why newness is important in rule $4 \mathrm{~A}$.

\section{REFERENCES}

[I] A. R. Anderson and N. D. Belnap, Jr., Entailment, The Logic of Relevance and Necessity, Vol. I, Princeton U. P., I975.

[2] R. T. Brady, "A Content Semantics for Quantified Relevant Logics II," Studia Logica, Vol. 48 (1989), pages 243-257.

[3] R. T. Brady, "The Gentzenization and Decidability of Rw," fournal of Philosophical Logic, Vol. 19 (1990), pages 35-73.

[4] R. T. Brady, "Gentzenization and Decidability of Some Contraction-less Relevant Logics," Fournal of Philosophical Logic, Vol. 20 (I991), pages $97-$ II 7 .

[5] R. T. Brady, "Simplified Gentzenizations for Contraction-less Logics," Logique et Analyse, special issue, Vol. 137-8 (1992), pages 45-67.

[6] K. Fine, "Models for Entailment," Fournal of Philosophical Logic, Vol. 3 (1974), pages 347-372.

[7] S. Giambrone, "Tw+ and RW+ are Decidable," Fournal of Philosophical Logic, Vol. I4 (1985), pages 235-254. 
[8] S. Giambone and R. K. Meyer, "Completeness and Conservative Extension Results for some Boolean Relevant Logics," Studia Logica, Vol. 48 (I989), pages I-I4.

[9] G. E. Hughes and M. J. Cresswell, An Introduction to Modal Logic, Methuen, 1968.

[ıo] G. Priest and R. Sylvan, "Simplified Semantics for Basic Relevant Logics," Fournal of Philosophical Logic, Vol. 2I (1992), pages 217-232.

[II] G. Restall, "Simplified Semantics for Relevant Logics (and Some of Their Rivals)," Fournal of Philosophical Logic, Vol. 22 (I993), pages 48I-5II.

[12] R. Routley, R. K. Meyer, V. Plumwood and R. T. Brady, Relevant Logics and their Rivals, Vol. I, Ridgeview, California, 1982.

[13] J. K. Slaney, "A Metacompleteness Theorem for Contraction-Free Relevant Logics," Studia Logica, Vol. 43 (1984), pages 159-I68.

[I4] J. K. Slaney, "Reduced Models for Relevant Logics Without w I," Notre Dame Fournal of Formal Logic, Vol. 28 (1987), pages 395-407.

[15] A. Urquhart, "The Undecidability of Entailment and Relevant Implication," Fournal of Symbolic Logic, Vol. 49 (I984), pages I059-IO73. 
The Australasian fournal of Logic (ISSN I448-5052) disseminates articles that significantly advance the study of logic, in its mathematical, philosohpical or computational guises. The scope of the journal includes all areas of logic, both pure and applied to topics in philosophy, mathematics, computation, linguistics and the other sciences.

Articles appearing in the journal have been carefully and critically refereed under the responsibility of members of the Editorial Board. Only papers judged to be both significant and excellent are accepted for publication.

The journal is freely available at the journal website at

$$
\text { http://www.philosophy.unimelb.edu.au/ajl/ }
$$

All issues of the journal are archived electronically at the journal website.

SUBSCRIPTIONS Individuals may subscribe to the journal by sending an email, including a full name, and email address and a postal address to the managing editor at ajl-editors@unimelb.edu.au Subscribers will receive email abstracts of accepted papers to an address of their choice. For institutional subscription, please email the managing editor at ajl-editors@unimelb.edu.au

Complete published papers may be downloaded at the journal's website at http: //www.philosophy.unimelb.edu.au/ajl/ The journal currently publishes in pdf format.

Submission The journal accepts submissions of papers electronically. To submit an article for publication, send the $\mathrm{LT}_{\mathrm{E}} \mathrm{X}$ source of a submission to a member of the editorial board. For a current list of the editorial board, consult the website.

The copyright of each article remains with the author or authors of that article. 